Check for updates

Cite this: RSC Adv., 2018, 8, 13789

Received 29th January 2018

Accepted 3rd April 2018

DOI: $10.1039 / c 8 r a 00907 d$

rsc.li/rsc-advances

\title{
Nanodiamond-based layer-by-layer nanohybrids mediate targeted delivery of miR-34a for triple negative breast cancer therapy
}

\author{
Yang Xia, $\uparrow$ Xiongwei Deng, $\uparrow$ Minjun Cao, Sha Liu, Xiaofei Zhang, Xiangqian Xiao, \\ Sisi Shen, Qin $\mathrm{Hu}^{*}$ and Wang Sheng (D) *
}

Triple-negative breast cancer (TNBC) is the most lethal subtype of breast cancer and significantly associated with poor prognosis and high risk of recurrence. miR-34a has been identified as a potent tumor suppressor whose expression is dramatically downregulated in TNBC. Currently, rectification of miRNA abnormality serves as a novel tumor therapeutic strategy. miR-34a is thus used as powerful antitumor agent for miRNA-based therapy against TNBC. However, miRNA-based antitumor therapy is challenged by effective targeted delivery of miRNA. In the present study, nanodiamond (ND), protamine (PS) and folic acid (FA) were used to construct ND-based layer-by-layer nanohybrids through a self-assembly approach for targeted miR-34a delivery in TNBC cells and xenograft TNBC tumors. We found that the targeted delivery of miR-34a remarkably suppressed cell proliferation, migration and induced the apoptosis of TNBC cells in vitro and inhibited tumor growth in vivo via down-regulating Fra-1 expression. The data suggest a great potential of ND-based nanohybrids for targeted intratumoral delivery of miR34a for TNBC therapy.

\section{Introduction}

Breast cancer is one of the most common cancers and a leading cause of cancer-related death in women worldwide. Triplenegative breast cancer (TNBC) represents a unique heterogeneous subtype that is defined by negative expression of estrogen receptor (ER), progesterone receptor (PR) and human epidermal growth factor receptor 2 (HER2). TNBC accounts for approximately $15-20 \%$ of all diagnosed breast cancer and is more commonly diagnosed in younger women. TNBC is frequently associated with poor prognosis, aggressive phenotype and high rate of recurrence due to lack of therapeutic targets. ${ }^{1-3}$

Recent discoveries have revealed that a number of microRNAs (miRNA) including miR-21, miR-34a, miR-215, miR-155, miR-30a, miR-9-3p and miR-143-5p were dysregulated in TNBC tissues and cells. ${ }^{4,5}$ miRNAs are conserved small noncoding RNAs that could function either as tumor suppressor or oncogene in tumorigenesis. The aberrant expression of miRNAs is highly associated with tumor cell proliferation, metastasis and is positively correlated with poor prognosis of TNBC. Moreover, accumulated evidences have revealed miRNAs as potential prognostic biomarkers in TNBC. $^{6}$ miR-34a,

College of Life Science and Bioengineering, Beijing University of Technology, 100 Pingleyuan, Chaoyang District, Beijing, P. R. China. E-mail: shengwang@bjut.edu. cn; hq07616@bjut.edu.cn; Fax: +86-10-67392780; Tel: +86-10-67391668; +86-1067392780

$\dagger$ Both authors contributed equally to this work. a member of the miRNA-34 family that is regulated by p53, has been found significantly down-regulated in TNBCs and correlated with poor survival rate. ${ }^{7,8}$ More importantly, miR-34a acts as a powerful tumor suppressor. The rectification of miRNA-34a abnormality using miRNA mimic elicited potent anti-tumor effects by inducing cell apoptosis and inhibiting cell proliferation and migration in TNBC models. ${ }^{9}$ Thus, antagonizing oncogenic miRNAs or overexpressing tumor suppressive miRNAs represent attractive therapeutic approaches for tumor therapy.$^{10}$ In the present study, miR-34a was engaged as potent tumor suppressor for miRNA-directed gene therapy against TNBC. However, there are several remaining unsolved issues for miRNA-based tumor therapy, one of the challenges is targeted tumor delivery.

Nanohybrids have been well recognized as efficient carriers to encapsulate multiple therapeutic agents for cancer diagnosis and therapy. In the past decades, positive charged nanocarriers, such as polymers, polysaccharides and liposomes have been developed to encapsulate negative charged miRNAs, and the miRNAs are protected from enzymatic degradation and immune surveillance before uptaken by targeted tumor cells. ${ }^{11}$ In the previous studies, we have demonstrated the effective selfassembly and intracellular delivery of miRNA-34a using hyaluronic acid (HA)-chitosan (CS) nanohybrids (NPs) and codelivery of miRNA-34a with DOX using hyaluronic acid (HA)/ protamine sulfate (PS) interpolyelectrolyte complexes (HPIPECs). Both of them exhibited powerful anti-tumor effects in TNBC in vitro and in vivo. ${ }^{12,13}$ 
Folate receptors (FR) are a family of membrane proteins that bind to folate with high affinity and regulate its delivery into cells. Previous studies have identified the effect of FRs on cell proliferation. ${ }^{\mathbf{1 4}}$ More importantly, the overexpression of FRs was detected in various carcinomas like ovarian, lung, kidney, colon and breast cancer. Recently, folic acid was conjugated on the surface of nanohybrids as novel approach for targeted intratumoral drug delivery with numerous advantages, such as high selectivity to FR-positive cells, low cytotoxicity, stability and enhanced tumor permeability. ${ }^{15-19}$ Nanodiamonds (NDs) are water-dispersible carbon-based nanoparticles with sizes varied between 2 and $8 \mathrm{~nm}$, which exhibit extraordinary biocompatibility and are suitable for various high-affinity binding of therapeutics. $^{20}$ In the present study, FA and PS were used to form layer-by layer ND-based nanohybrids via self-assembly approach for targeted delivery of miR-34a in TNBC cells and xenograft TNBC tumors. The anti-tumor effects of miR-34a delivered by ND-based layer-by layer NPs were evaluated in vitro and in vivo in xenograft animal model.

\section{Materials and methods}

\section{Materials}

The acid-oxidated ND gel $(15 \% \mathrm{w} / \mathrm{v}$ in water) was purchased from the NanoCarbon Research Institute Ltd. As-received NDs were dispersed in deionized water and diluted to $1 \mathrm{mg} \mathrm{ml} \mathrm{ml}^{-1}$ after overnight ultrasonication. The protamine sulfate (PS) and folate acid (FA) were supplied by Sigma Aldrich and were dissolved in nuclease-free water and phosphate buffered saline (PBS, pH8.0) respectively, at the concentration of $10 \mathrm{mg} \mathrm{ml}^{-1}$. MicroRNA-34a (miR-34a) mimics, Cy3-labeled miR-34a (Cy3miR-34a) mimics and scramble microRNA (NC miRNA) mimics were obtained from RiboBio Co. Ltd. RMPI 1640 medium, PBS, fetal bovine serum (FBS), trypsin/EDTA solution and penicillin/streptomycin were purchased from Gibco BRL. All other chemicals and solvents were of the highest grade commercially available.

\section{Preparation of PS@NDs, miR-34a/PS@NDs, PS/miR-34a/ PS@NDs and FA/PS/miR-34a/PS@NDs}

To prepare protamine sulfate-functionalized nanodiamonds (PS@NDs), the ND solution was mixed with PS at a $1: 20$ mass ratio and the resulting mixture was agitated at room temperature for $15 \mathrm{~min}$. Unbound protamines were then removed by extensive wash. The PS@NDs pellet was re-dispersed in water and the concentration was determined based on the lyophilized weight of $1 \mathrm{ml}$ solution. To prepare miR-34a-loaded nanohybrids (miR-34a/PS@NDs), miR-34a mimics were incubated with PS@NDs at a $1: 4$ mass ratio and incubated for $15 \mathrm{~min}$ at room temperature before measurement. The resulting mixture was then slowly mixed with the PS solution and further isolated by centrifugation to produce PS/miR-34a/PS@NDs. ${ }^{18}$ For the preparation of FA/PS/miR-34a/PS@NDs, PS/miR-34a/PS@NDs solution was slowly mixed with over-dose FA solution followed by centrifugation and resuspension.

\section{Characterization of the nanohybrids}

The average size and zeta potential of the PS@NDs, miR-34a/ PS@NDs, PS/miR-34a/PS@NDs and FA/PS/miR-34a/PS@NDs were determined by dynamic light scattering (DLS) using a Zetasizer Nano ZS (Malvern). The samples were diluted in distilled water and the measurements were carried out at $633 \mathrm{~nm}$ with a constant angle of $90^{\circ}$. Each experiment was performed in triplicate. Morphological examination of the four nanohybrids were performed by transmission electron microscopy (TEM) (EM-200CX, JEOL Ltd.). The samples were stained with $1 \%(\mathrm{w} / \mathrm{v})$ phosphotungstic acid for $10 \mathrm{~s}$, immobilized on copper grids and dried overnight for viewing in the TEM.

\section{Gel electrophoresis and serum stability assay}

The serum stability of FA/PS/miR-34a/PS@NDs were analyzed by $2 \%$ agarose gel electrophoresis. The gels were freshly prepared with $2 \%$ agarose in tris-acetate-ethylenediaminetetraacetic acid (EDTA) buffer containing $0.5 \mu \mathrm{g}$ $\mathrm{ml}^{-1}$ GelREDTM (Biotium). The FA/PS/miR-34a/PS@NDs were mixed with FBS at serum concentration of $50 \%$. After the incubation for the indicated times at $37{ }^{\circ} \mathrm{C}$, the same amount $(15 \mu \mathrm{l})$ of each sample were loaded onto agarose gel for electrophoresis at $110 \mathrm{~V}$ for $10 \mathrm{~min}$ and imaged using gel imager system (Bio-rad).

\section{Cell culture and cellular uptake}

The human triple-negative breast cancer cell lines MDA-MB-231 (kindly provided by Prof. Y. Wu from National Center for Nanoscience and Technology, Beijing, China) was cultured in RPMI-1640 medium supplemented with 10\% fetal bovine serum (FBS) (Gibco) and incubated in a humidified atmosphere with $5 \% \mathrm{CO}_{2}$ at $37{ }^{\circ} \mathrm{C}$. Cellular uptake of FA/PS/miR-34a/ PS@NDs carrying Cy3-labeled miR-34a was evaluated using flow cytometry (FACSAria Cell Sorter, Becton Dickinson BD) and confocal laser scanning microscopy (CLSM, Carl Zeiss). In the flow cytometry assay, FA/PS/miR-34a/PS@NDs, miR-34a/ PS@NDs, Block-FA-miR-34a and Ctr groups received treatments as described above. Then the cells were harvested and resuspended in $1 \mathrm{ml}$ PBS for FACS analysis (Cy3: $\lambda_{\mathrm{Ex}}=570 \mathrm{~nm}$, $\lambda_{\mathrm{Em}}=660 \mathrm{~nm}$ ). In the confocal microscopy assay, MDA-MB-231 cells were harvested and seeded onto a borosilicate chambered cover glass (Invitrogen) at a density of $1 \times 10^{5}$ cells per well and cultured for $12 \mathrm{~h}$ at $37^{\circ} \mathrm{C}$ before the transfection. Cells in BlockFA-miR-34a group were blocked with over-dose FA solution for 6 h. The cells were then washed three times with PBS and incubated with FA/PS/miR-34a/PS@NDs (100 nM of miR-34a) in serum-free medium for $6 \mathrm{~h}$ at $37{ }^{\circ} \mathrm{C}$, followed by a medium change to fresh complete medium. Eighteen hours later, the cells were washed with PBS and fixed with $4 \%$ formaldehyde for $30 \mathrm{~min}$ at room temperature. DAPI (Beyotime) was used to stain the cell nuclei.

\section{Cell viability and apoptosis assay}

MDA-MB-231 cells were seeded in 96-well plates at a density of 3 $\times 10^{3}$ cells per well and incubated at $37^{\circ} \mathrm{C}$ with $5 \% \mathrm{CO}_{2}$ for $12 \mathrm{~h}$. 
For FA-blocking group (FA-Block-miR-34a), cells were pretreated with an overdose of FA solution and cells in the other groups were treated with equal amount of PBS. After $6 \mathrm{~h}$ of incubation, medium was removed and replaced with serum-free medium containing FA/PS/miR-34a/PS@NDs or FA/PS/NC miR/PS@ND (scramble-miR control). The cells without treatment were set as negative control. The culture media containing nanohybrids was then replaced by fresh complete RPMI-1640 (10\% FBS) after $6 \mathrm{~h}$ treatment. Cell viability was determined at indicated time points using CCK-8 assay kit (Dojindo) according to manufacturer's instructions. The percentage of the viable cells was calculated: viability $\%=\left(\mathrm{Ab}_{\text {test }}-\mathrm{A} \mathrm{b}_{\text {blank }}\right) /\left(\mathrm{Ab}_{\mathrm{ctr}}-\mathrm{Ab}_{\text {blank }}\right) \times$ $100 \%$ ( $\mathrm{Ab}$ is the absorbance at $450 \mathrm{~nm}$ ).

Annexin V/propidium iodide (PI) apoptosis detection kit (BD) was used for apoptosis assessment. MDA-MB-231 cells treatments with FA/PS/miR-34a/PS@NDs, FA/PS/NC-miR/ PS@NDs and FA-Block-miR-34a were the same as described in cell viability assay. After treatment of the nanohybrids for $6 \mathrm{~h}$, the cells were incubated in fresh RPMI-1640 (10\% FBS) at $37{ }^{\circ} \mathrm{C}$ with $5 \% \mathrm{CO}_{2}$ for $18 \mathrm{~h}$ and then were processed with serum starvation by exchanging the media with RPMI-1640 without FBS for $48 \mathrm{~h}$. The cells were carefully collected and processed by using annexin V/PI apoptosis detection kit according to the instructions. Each experiment was carried out in triplicate.

\section{Quantitative real-time PCR}

Quantitative real-time PCR (qRT-PCR) was applied to evaluate the expression level of miR-34a and Fra-1 gene. For the miR-34a detection, total RNA was extracted using Trizol reagent (Invitrogen). MicroRNA reverse transcription reactions were performed using Taqman ${ }^{\circledR}$ MicroRNA Reverse Transcription Kit (Applied Biosystems). qPCR was conducted using TaqMan ${ }^{\circledR}$ miRNA Expression assays (ID 000426, Invitrogen) and Taqman ${ }^{\circledR}$ Universal Master Mix II (Invitrogen) according to the manufacturer's protocol. The thermocycling conditions were as follows: $95^{\circ} \mathrm{C}$ for $10 \mathrm{~min}, 40$ cycles at $95^{\circ} \mathrm{C}$ for $15 \mathrm{~s}$ and $60{ }^{\circ} \mathrm{C}$ for $1 \mathrm{~min}$. U6 was used as normalization control.

To evaluate the Fra-1 gene expression level, ImProm-II ${ }^{\mathrm{TM}}$ reverse transcription system (Promega) was used to synthesize the first-strand cDNA and Brilliant II SYBR qPCR master mix (Stratagene) was used to perform qRT-PCR $\left(50{ }^{\circ} \mathrm{C}\right.$ for $2 \mathrm{~min}$, $95{ }^{\circ} \mathrm{C}$ for $2 \mathrm{~min}, 40$ cycles at $95{ }^{\circ} \mathrm{C}$ for $15 \mathrm{~s}$ and $55^{\circ} \mathrm{C}$ for $1 \mathrm{~min}$, followed by 1 cycle for melting curve at $60{ }^{\circ} \mathrm{C}$ to $95^{\circ} \mathrm{C}$ ). GAPDH was used as an endogenous control for normalization.

\section{Western blot assay}

After treatments, MDA-MB-231 cells were harvested and lysed by RIPA lysis buffer. The protein samples from Ctr, FA-Block-miR34a and FA/PS/miR-34a/FA/PS@NDs groups were loaded onto SDS-PAGE gel in equal amounts for electrophoresis. Then the proteins were transferred to PVDF membranes (Millipore) followed by blocking with 5\% non-fat dry milk in TBS for $4 \mathrm{~h}$ at room temperature. The anti-Fra-1 antibody (Santa cruz) and anti-GAPDH antibody (RD) were used to probe the immunoblots overnight at $4{ }^{\circ} \mathrm{C}$. The membranes were then washed with TBST solution and incubated with fluorescent dyes-labeled secondary antibody (KPL) for $50 \mathrm{~min}$ at room temperature. The blotting images were acquired by the Odyssey CLx infrared imaging system (Li-COR).

\section{Transwell migration assay}

MDA-MB-231 cells were seeded and treated with nanohybrids as described above. After $18 \mathrm{~h}$ of incubation with fresh RPMI-1640 (10\% FBS), cells were harvested and plated into the upper chambers of the Transwell insert (Corning) in serum-free medium. Medium containing 20\% FBS was added to the lower chamber. After incubation for $24 \mathrm{~h}$, the cells on the upper surface were removed and cells migrated to the lower surface were fixed and stained with $0.2 \%$ crystal violet for $10 \mathrm{~min}$. Migrated cells were counted in five randomly-selected fields using bright-field microscopy (Zeiss). The cell migration rate was calculated: relative migration rate $(\%)=$ (number of migrated cells with treatment)/(number of migrated cells without treatment) $\times 100 \%$. Each batch was carried out in triplicate.

\section{In vivo anti-tumor experiments}

For in vivo experiments, female athymic nude Balb/c mice (4-6 weeks) were obtained from Beijing Vital River Laboratory Animal Technology Co. Ltd and all animals were kept under specific pathogen-free (SPF) conditions. Mice were randomly assigned to 4 groups and injected subcutaneously into the right flank with MDA-MB-231 cells $\left(5 \times 10^{6}\right.$ cells suspended in $100 \mu \mathrm{l}$ PBS solution for each mouse). When the tumor volumes reached approximately $0.1 \mathrm{~cm}^{3}$, mice were intravenously injected with PBS, FA/PS/NC-miR/PS@NDs (NC-miRNA: $2 \mathrm{mg} \mathrm{kg}^{-1}$ ), FA/PS/miR-34a/PS@NDs (miR-34a: $2 \mathrm{mg} \mathrm{kg}^{-1}$ ), or FA/PS/miR34a/PS@NDs (intratumor injection, miR-34a: $2 \mathrm{mg} \mathrm{kg}^{-1}$ ) every 2 days for 7 times. Tumor volume was measured before every injection and calculated using the formula: $V=L \times W^{2} / 2$, where $L$ is the longest diameter and $W$ is the shortest diameter. All the animals were sacrificed to extracting tumors at the end of the experiments. The tumor tissues were extracted and fixed by $4 \%$ polyoxymethylene, and then the paraffin embedding and hematoxylin and eosin staining (H\&E staining) were performed. All experimental protocols involving animal study were in accordance with the "Guidelines for the Care and Use of Laboratory Animals" published by the National Institutes of Health (NIH publication no. 85-23, revised 1996) and were approved by the Institutional Animal Care and Use Committee (IACUC) of Institute of Process Engineering, Chinese Academy of Science.

\section{In vivo imaging and biodistribution analysis}

The in vivo images and biodistribution of FA/PS/Cy3-miR-34a/ PS@NDs after intravenous injection were carried out using the ex/in vivo imaging system (CRi, Woburn, MA). FA/PS/Cy3-miR34a/PS@NDs $(100 \mu \mathrm{g})$ was administrated to the tumor-bearing mice via tail vein injection when the tumor volumes reached approximately $0.3 \mathrm{~cm}^{3}$. The mice injected with PS/Cy3-miR34a/PS@NDs were set as comparison. The fluorescent signals of Cy3 were collected at $24 \mathrm{~h}$ and $48 \mathrm{~h}$ post injection. At the end 
of the experiment, the mice were sacrificed, tumors and major organs were extracted for imaging.

\section{Statistical analysis}

Data were expressed as mean \pm standard deviation. One-way analysis of variance (ANOVA) was used to evaluate the statistical significance. A $P$ value of $<0.05$ was considered statistically significant different.

\section{Results}

\section{Preparation and characterization of nanohybrids}

We previously showed that PS was able to interact with oxidized NDs to form PS@ND hybrid through self-assembly approach (Fig. 1A). ${ }^{21}$ Dynamic light scattering (DLS) analysis revealed a positive charge of PS@ND hybrids upon the binding of PS on the surface of oxidized ND. Negatively charged miR-34a was then added and adsorbed on the positively charged surface of PS@ND hybrids through electrostatic interactions, which led to a negatively charged surface of miR-34a/PS@ND hybrids (Fig. 1B). PS was subsequently added and resulted in positively charged surface of PS/miR34a/PS@ND hybrids to form layer by layer nanohybrids.
Negatively charged FA was added to form the outermost layer, which led to a negatively charged surface of FA/PS/miR-34a/ PS@ND nanohybrids (Fig. 1B). As shown in Fig. 1C, the average hydrodynamic size of miR-34a/PS@NDs was increased to $173 \mathrm{~nm}$ after adsorption of miR-34a on surface of PS@NDs, whose average size is $100 \mathrm{~nm}$. In addition, the average sizes of PS/miR-34a/PS@NDs increased to $202 \mathrm{~nm}$ compared to miR-34a/PS@NDs after addition of PS and the average size of FA/PS/miR-34a/PS@NDs nanohybrids reached $210 \mathrm{~nm}$ upon encapsulation of FA on surface of PS/miR-34a/ PS@NDs, indicating that the size of PS@ND-based nanohybrids was gradually increased after one-by-one addition of miR-34a, PS and FA (Fig. 1C).

The formation of PS@ND, miR-34a/PS@ND, PS/miR-34a/ PS@ND and FA/PS/miR-34a/PS@NDs nanohybrids was also structurally detected by transmission electron microscopy (TEM) imaging. As shown in Fig. 1D. The functionalized NDs, which showed well-defined atomic lattice fringes, were coated by amorphous materials composed of PS, miRNA and FA. It is worth noting that the TEM results were greatly different with the hydrodynamic size of DLS measurements. The DLS results reflected the clusters of the nanohybrids, while the TEM images reflected the regional structure of the nanohybrids.

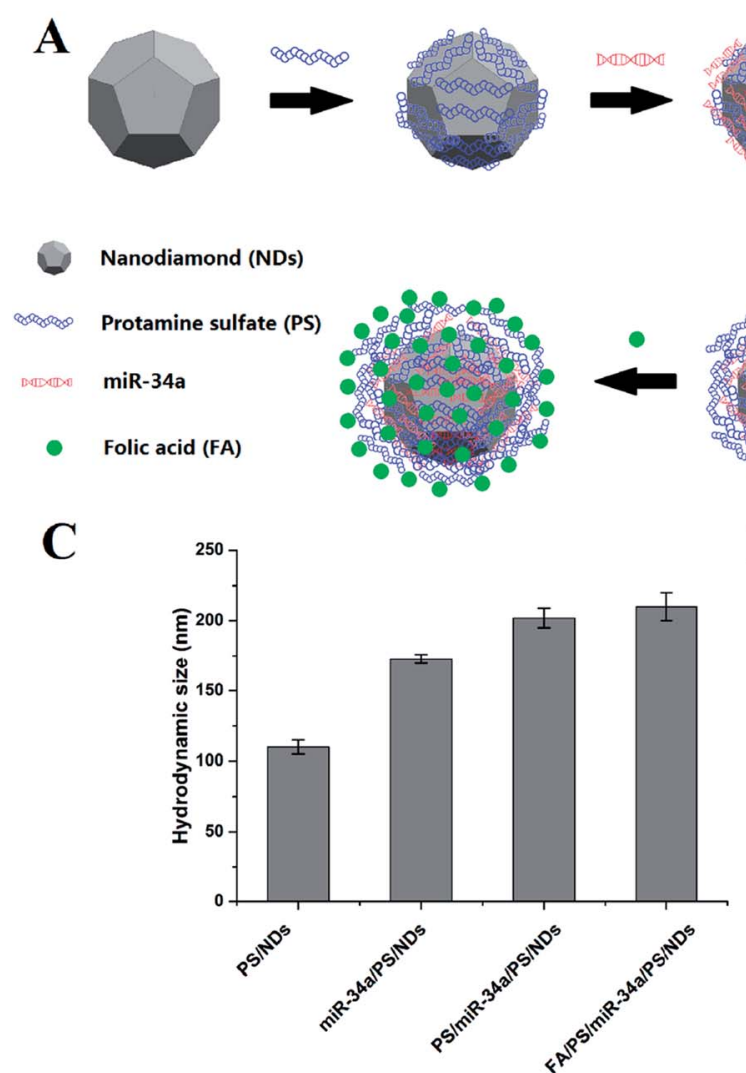

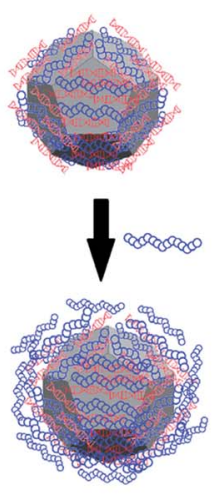

D
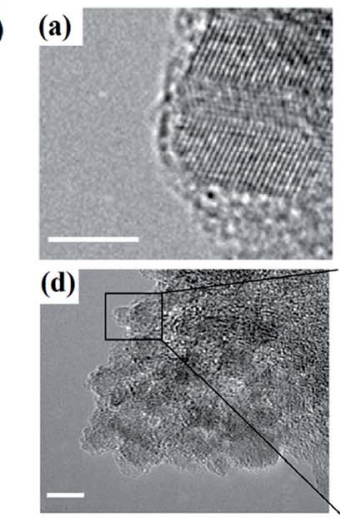

B
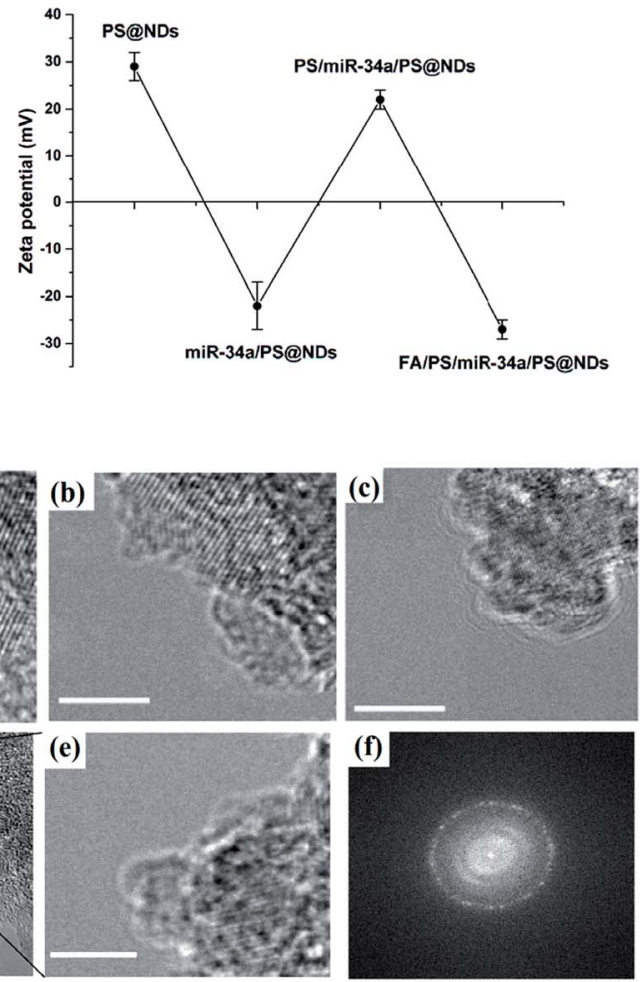

Fig. 1 Schematic illustration and characterization of nanohybrids. (A) Schematic illustration of the construction of FA/PS/miR-34a/PS@NDs through self-assembly approach. (B) Surface zeta potential of nanohybrids which was expressed as means \pm S.D $(n=3)$. (C) The size distribution of nanohybrids, which was expressed as means \pm S.D $(n=3)$. (D) TEM imaging. (a) PS/NDs, (b) miR-34a/PS@NDs, (c) PS/miR-34a/PS@NDs, (d) FA/ PS/miR-34a/PS@NDs. (e) Magnified TEM image of the marked part in (d). (f) The corresponding FFT pattern of FA/PS/miR-34a/PS@NDs. The scale bars represent $5 \mathrm{~nm}$. 
A

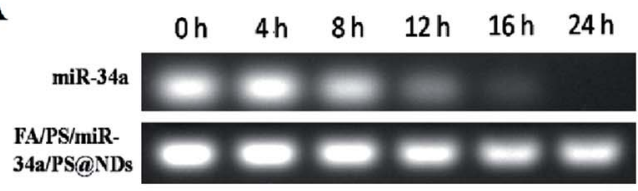

C

FA-Block-miR-34a

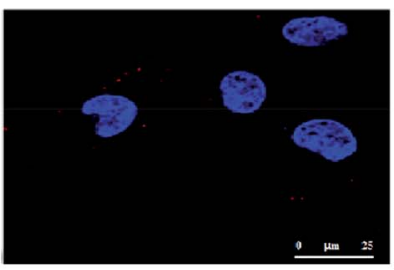

FA/PS/miR-34a/PS@NDs

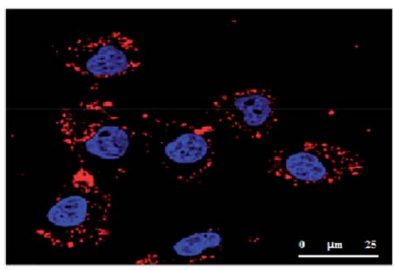

B

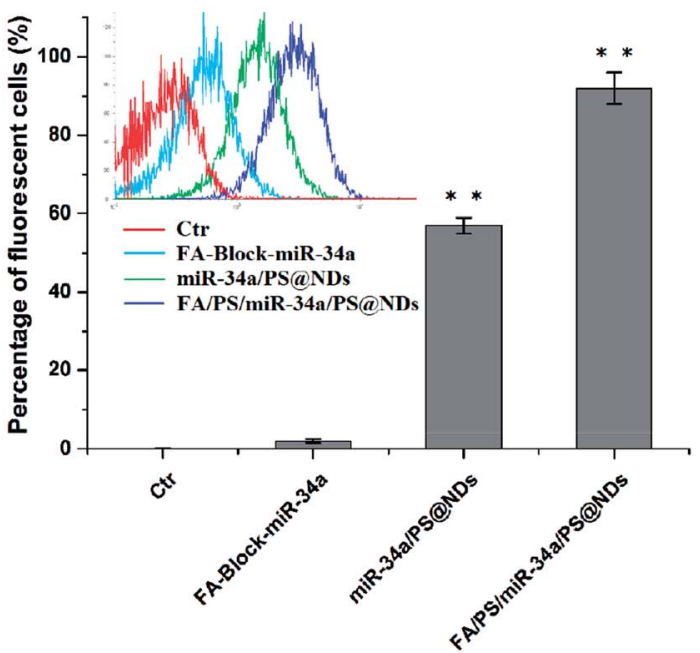

Fig. 2 Serum stability and cell uptake of nanohybrids. (A) The serum stability of nanohybrid-encapsulated miR-34a was evaluated by gel electrophoresis. Free miR-34a was served as control. (B) Cell uptake of Cy3-labeled miR-34a analyzed by flow cytometry. Data were presented as means \pm S.D $(n=3),{ }^{* *} p<0.01$. (C) Cellular uptake of Cy3-labeled miR-34a detected by confocal microscopy.

\section{Serum stability of miR-34a and cellular uptake}

Stability of miR-34a in serum was measured by gel electrophoresis. As shown in Fig. 2A, miR-34a encapsulated into FA/PS/ miR-34a/PS@NDs nanohybrids was stable in serum for up to $24 \mathrm{~h}$ whereas the naked miR-34a mimics band started to decrease after $8 \mathrm{~h}$ of incubation and drop to an undetectable value $24 \mathrm{~h}$ post-incubation, indicating that FA/PS/miR-34a/ PS@NDs nanohybrids prevent the degradation of miR-34a in serum. Nanohybrids-mediated cellular uptake of Cy3-labeled miR-34a was further evaluated by flow cytometry (FACS) analysis. We observed that signals of Cy3-labeled miR-34a was significantly stronger in FA/PS/miR-34a/PS@NDs nanohybridstreated cells than those treated by miR-34a/PS@ND nanohybrids. Moreover, pretreatment with folic acid reduced cell uptake of Cy3-labeled miR-34a (Fig. 2B). It was consistent with the data obtained in confocal laser scanning microscopy analysis, in which fluorescent signals were remarkably decreased in FA/PS/miR-34a/PS@NDs nanohybrids-treated cells after pretreatment with folic acid (Fig. 2C). The data suggested that FA attachment on nanohybrids surface played a critical role in efficient cell uptake of ND-based nanohybrids and significantly improved cell uptake of nanohybrids.

\section{Suppression of Fra-1 expression by miR-34a restoration}

The qRT-PCR assay was conducted to evaluate restoration level of miR-34a and expression of its target gene, Fra-1. Remarkable increase of miR-34a was observed in FA/PS/miR-34a/PS@NDs nanohybrids-treated cells compared to the untreated control cells and FA pretreatment dramatically decreased miR-34a level (Fig. 3A), indicating that miR-34a was efficiently delivered into the cells by nanohybrids via FA receptor. Fra-1 expression was also significantly reduced upon nanohybrids treatment, which
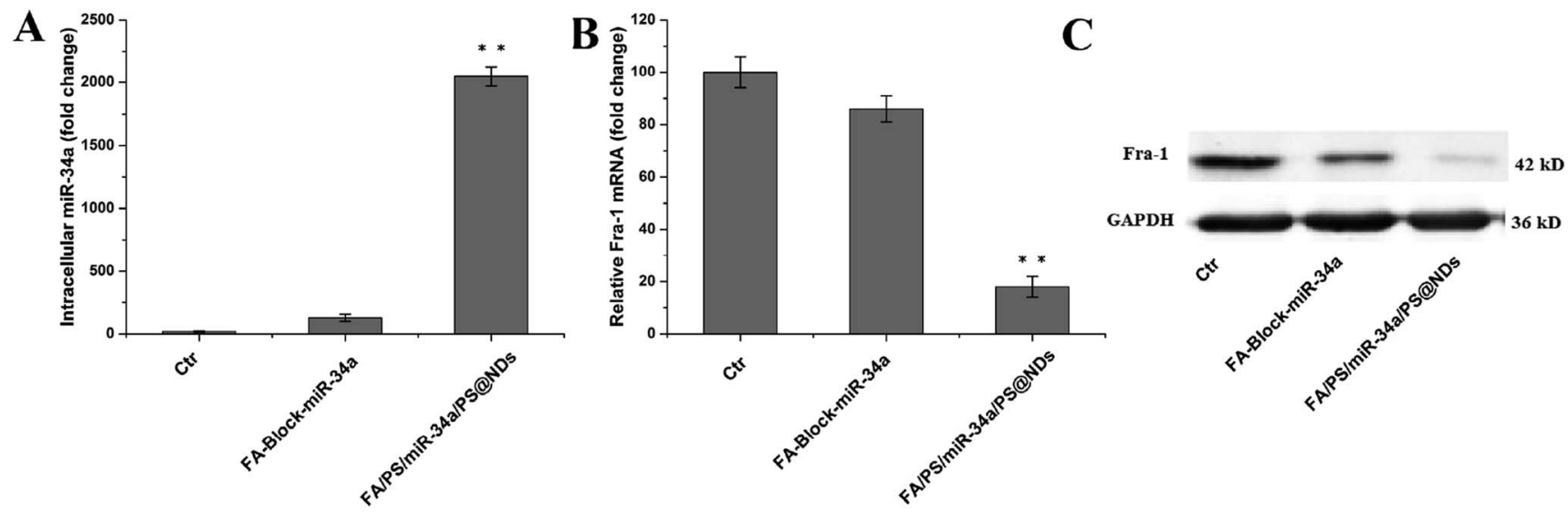

Fig. 3 (A) Intracellular delivery of miR-34a and analysis of Fra-1 expression. (A) miR-34a level was detected by RT-PCR. (B) Fra-1 expression was quantitatively measured by RT-PCR. Data were presented as means \pm S.D $(n=3)$, ** $p<0.01$. (C) Fra-1 expression was analyzed by western blot. 
A

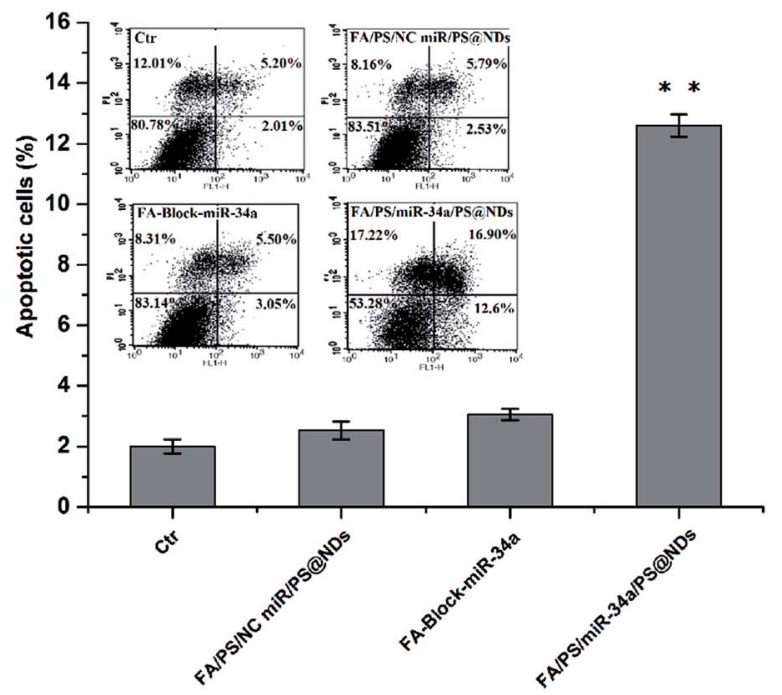

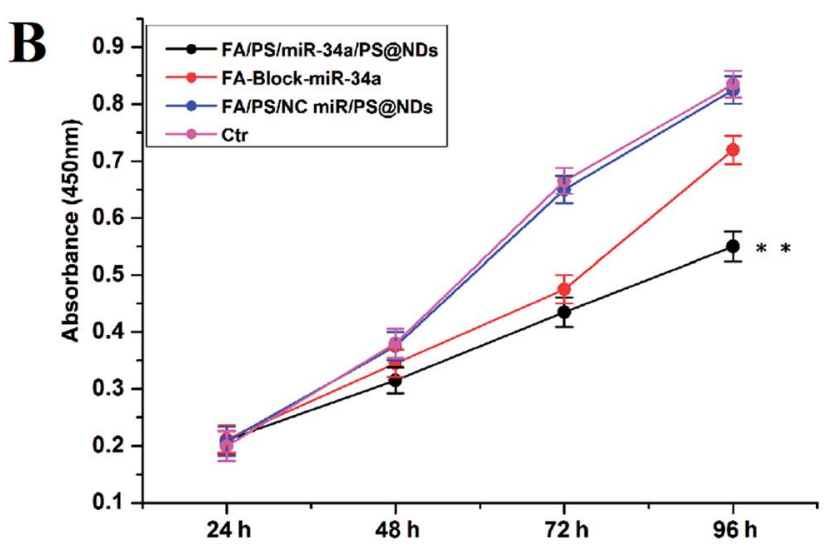

Fig. 4 Apoptosis and proliferation analysis. (A) Cell apoptosis was measured by FACS analysis using annexin V and PI staining. Percentage of apoptotic cell was calculated as ratio of apoptotic cells to total cell numbers. (B) Cell proliferation was quantitatively evaluated by $C C K-8$ assay. Data were presented as means \pm S.D $(n=3), * * p<0.01$.

was reversed by FA pretreatment, suggesting that Fra-1 expression is particularly down-regulated by intracellular delivery of miR-34a (Fig. 3B). Fra-1 expression was also confirmed by western blot assay (Fig. 3C).

\section{Cell viability, apoptosis and migration analysis}

The apoptosis, proliferation and migration of MDA-MB-231 cells were further investigated upon FA/PS/miR-34a/PS@NDs treatment. Untreated cells and cells treated by FA/PS/ PS@NDs-encapsulated scramble miRNA were used as control. As shown in Fig. 4A, increased apoptosis was particularly found in FA/PS/miR-34a/PS@NDs-treated MDA-MB-231 cells compared to those pretreated by FA and control samples. Moreover, cell growth was dramatically inhibited by FA/PS/miR34a/PS@NDs nanohybrids treatment compared to FApretreated cells and control samples (Fig. 4B). In addition, the treatment of FA/PS/miR-34a/PS@NDs nanohybrids remarkably suppressed MDA-MB-231 cell migration compared to control samples, FA-pretreatment reversely diminished nanohybridsmediated migration suppression (Fig. 5A and B), indicating that miR-34a was efficiently delivered into MDA-MB-231 cells by FA/PS/miR-34a/PS@NDs nanohybrids via a FA-associated pathway and exhibited anti-tumor effects by inducing the apoptosis and suppressing the proliferation and migration of MDA-MB-231 cells.

\section{In vivo anti-tumor efficacy}

The in vivo anti-tumor activity of FA/PS/miR-34a/PS@NDs was evaluated in MDA-MB-231 xenograft mouse model. Untreated mice and mice treated by PBS solution and scrambled miRNA were used as controls. Tumor volumes were measured 7 times for 14 days. As shown in Fig. 6A, intratumoral and intravenous treatment by FA/PS/miR-34a/PS@NDs nanohybrids significantly inhibited tumor growth compared with control groups. The most potent inhibition was observed in intratumoral administration group (Fig. 6B). The expression of miR-34a and Fra-1 in tumor tissues were subsequently analyzed by qRT-PCR. Increased miR-34a and reduced Fra-1 expression were obtained in groups with intratumoral and intravenous administration of FA/PS/miR-34a/PS@NDs nanohybrids compared to the control groups (Fig. 6C and D), indicating miR-34a was efficiently delivered into xenograft tumors by FA/PS/miR-34a/ PS@NDs nanohybrids and led to suppressed expression of Fra-1. Fra-1 expression was also detected by western-blot, the result was consistent with qRT-PCR data (Fig. 6E). The H\&E staining of tumor samples (Fig. $6 \mathrm{~F}$ ) demonstrated that FA/PS/ miR-34a/PS@NDs treatment efficiently triggered cell apoptosis in tumor tissues. Furthermore, FA-mediated targeted delivery of nanohybrids was visualized using in vivo imaging system. As shown in Fig. 7A, fluorescence signals were significantly accumulated at tumor site $48 \mathrm{~h}$ after intravenous injection of FA/PS/ miR-34a/PS@NDs nanohybrids, whereas distribution of PS/ miR-34a/PS@NDs nanohybrids without FA attachment exhibited less concentrated tendency. The FA attachment remarkably enhanced the efficiency of tumor-targeted delivery of miR-34a. The data indicated that targeted tumor delivery of nanohybrids was mediated by FA attachment on hybrid surface. The fluorescence imaging captured from tumor tissues, heart, liver, lung, spleen, kidney and intestines showed that the nanoparticle was accumulated in tumor tissue other than normal organs (Fig. 7B).

\section{Discussion}

miR-34a, a member of highly conserved miR-34 family, acts as a potent tumor suppressor in various types of cancers, including lung, pancreatic, liver and breast cancer. ${ }^{22-25}$ The downregulation of miR-34a has not only been identified as diagnostic and prognostic biomarker in breast cancer, but also 
A

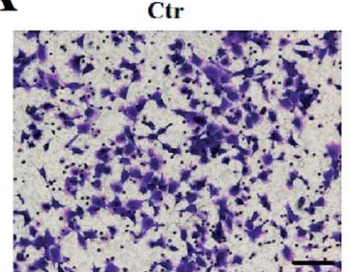
FA-Block-miR-34a

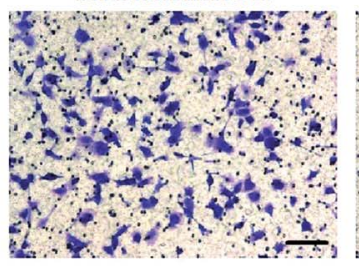

FA/PS/NC miR/PS@NDs

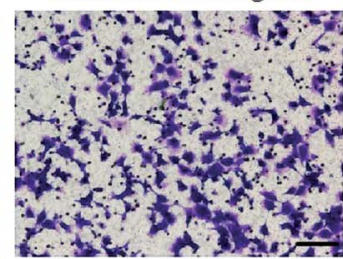

FA/PS/miR-34a/PS@NDs

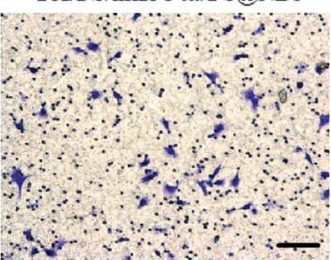

B

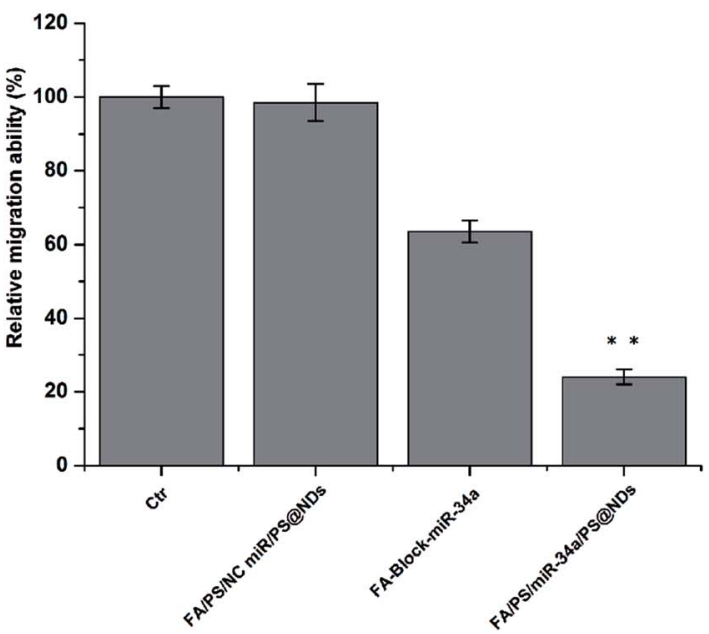

Fig. 5 Cell migration. (A) Cell migration was measured by transwell assay. Scale bar: $100 \mu \mathrm{m}$. (B) Quantitative determination of cell migration performed in triplicate presented as mean $\pm \mathrm{SD}, * * p<0.01$.

served as an effective therapeutic target of breast cancer. ${ }^{26}$ Being the first miRNA that has reached clinical trial for human liver cancer treatment, miR-34a has attracted intense attention and been extensively investigated in cancer treatment via gene replacement strategy. ${ }^{27}$

The FA/PS/miR-34a/PS@NDs nanohybrids were formed via an easy layer-by-layer assembly process. Negatively charged miR-34a was immobilized on the surface of positively charged protamine sulfate-functionalized nanodiamond nanohybrids. Protamine is an arginine-rich polycationic protein and approved by FDA for the treatment of heparin overdosage. In our previous studies, we reported that PS@NDs nanohybrids can be used as a safe platform for miRNA delivery for esophageal carcinoma treatment. ${ }^{20}$ In the present study, FA was introduced into the miR-34a/PS@NDs nanohybrids for tumortargeted delivery of miR-34a. FA has a high binding affinity to folate receptor, which is a GPI-anchored glycoprotein overexpressed in multiple cancer types, including lung, breast, kidney, ovarian cancers. ${ }^{28}$ The folate receptor $\alpha$ was found significantly upregulated in TNBCs and overexpression of folate receptor was highly associated with poor prognosis in breast cancer patients. $^{29-31}$ Thus, anti-folate receptor strategy might have advantage of specifically targeting TNBCs. We found a significant increase in cell uptake of FA/PS/miR-34a/PS@NDs nanohybrids, which can be reduced by FA pretreatment. The data suggest an important role of FA in intracellular delivery of nanohybrids in tumor cells.
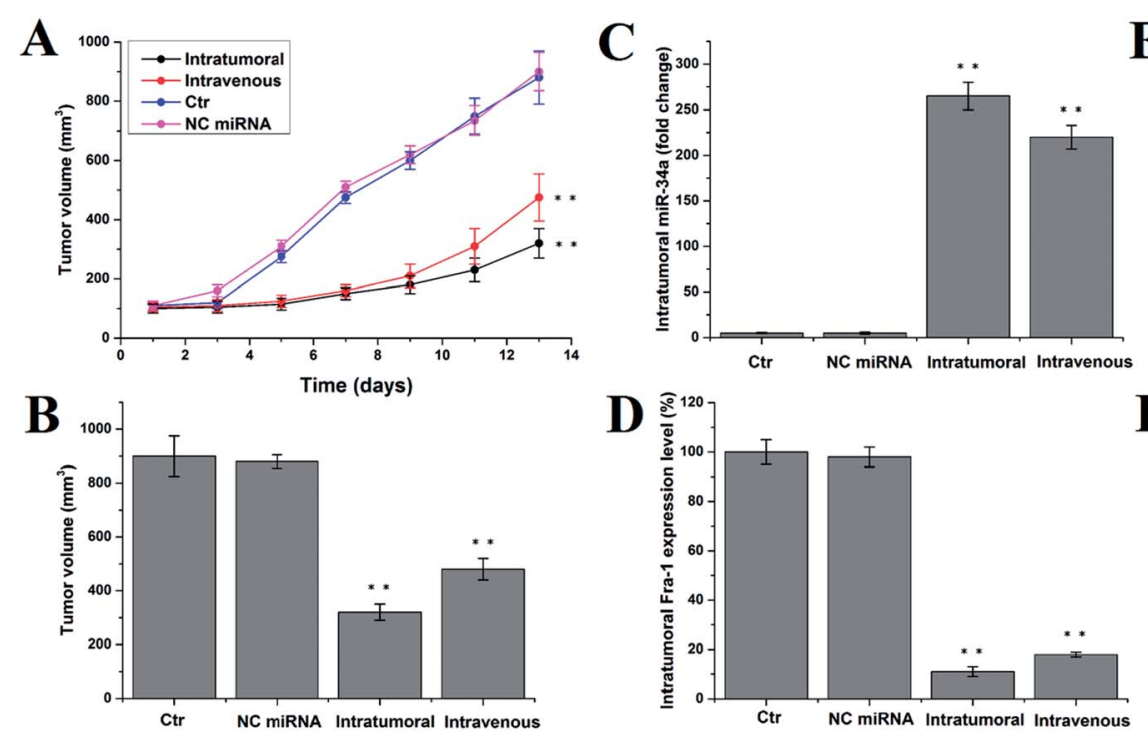

E

D

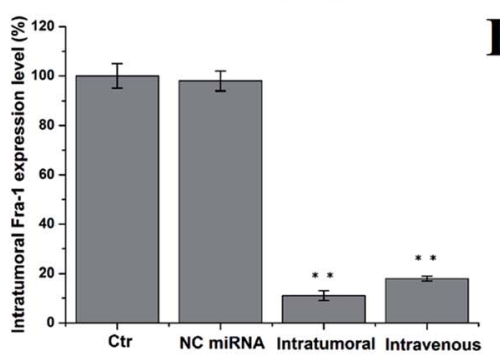

F

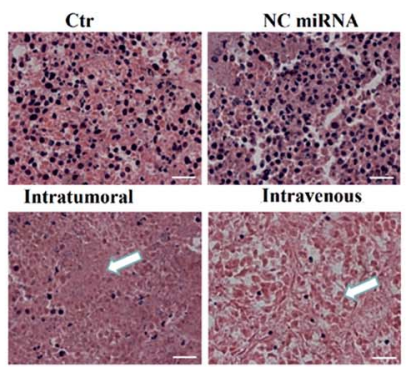

Fig. 6 In vivo antitumor activity. (A) Tumor volumes were measured at shown time-points and demonstrated as average of duplicate measurements with standard error bars $(n=5)$ for each group, $* * p<0.01$. (B) The tumors volumes were calculated at time of sacrifice and presented as mean $\pm \mathrm{SD}, * * p<0.01$. (C) miR-34a level was quantitatively measured by RT-PCR and shown as mean $\pm \mathrm{SD}, * * p<0.01$. (D) Expression of Fra-1 was quantitatively analyzed by RT-PCR and demonstrated as mean \pm SD, **p<0.01. (E) Expression of Fra-1 was detected by western blot. (F) Representative data of H\&E staining of tumor tissues. Scale bar: $50 \mu \mathrm{m}$. 
A

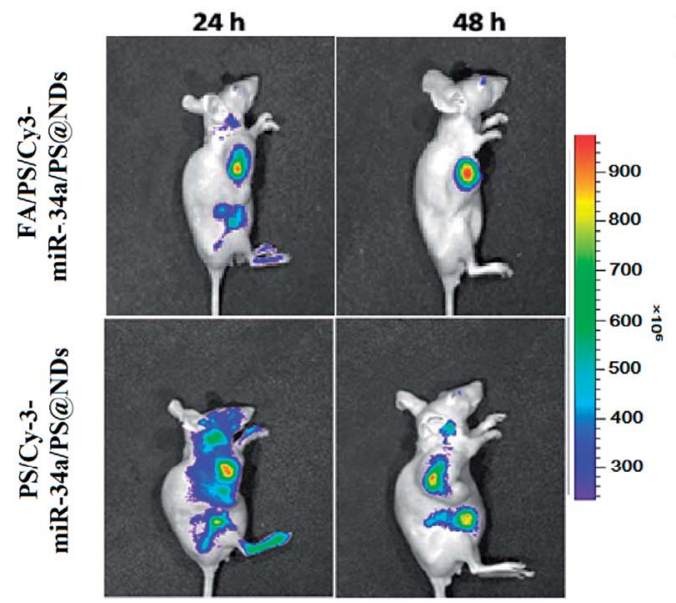

B

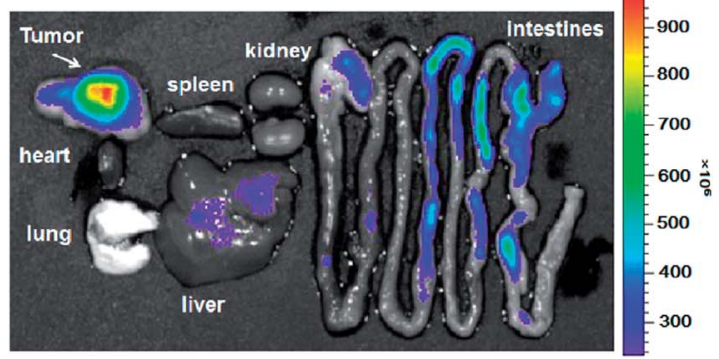

Fig. 7 Real-time in vivo fluorescence imaging. (A) In vivo fluorescence images were captured in real-time at indicated time points after nanohybrids injection through tail vein. (B) Tumor tissues, liver, lung, spleen, kidney, heart and intestine were isolated at $48 \mathrm{~h}$ post-injection for fluorescence imaging analysis.

Fra-1 is an AP-1 transcription factor subunit and plays an important role in tumorigenesis. ${ }^{32}$ Overexpression of Fra-1 in TNBCs promoted cell proliferation and motility. ${ }^{33}$ Our finding suggested that nanohybrids-mediated miR-34a delivery is efficient to halt MDA-MB-231 cell growth and migration partially via Fra-1 silencing. The anti-tumor effects of FA/PS/miR-34a/ PS@NDs were further confirmed using MDA-MB-231 xenograft mouse model, in which FA/PS/miR-34a/PS@NDs suppressed tumor growth by inducing the apoptosis of tumor cells. The in vivo imaging analysis showed that FA played a key role in tumortargeted delivery of miRNA mediated by ND-based nanohybrids. The data suggest that the ND-based layer-by-layer nanohybrids has a great potential to be a safe and efficient platform for tumor-targeted delivery of tumor suppressive miRNA for miRNA-based tumor therapy.

\section{Conclusions}

In summary, nanohybrids FA/PS/miR-34a/PS@NDs were developed as an efficient tumor-targeting miRNAs delivery system. Systemic and intratumoral delivery of miR-34a significantly inhibited tumor growth and inducing cell apoptosis via downregulating Fra-1 expression. The FA functionalized ND-based nanocomplexes hold a great promise for tumor-targeted therapy based on miRNA silencing strategy.

\section{Conflicts of interest}

The authors declare no competing financial interest.

\section{Acknowledgements}

The authors gratefully acknowledged that this work was financially supported by the National Natural Science Foundation (NSFC31440059, NSFC31770999 and NSFC81272453), and Beijing Advanced Innovation Center for Structural Biology (ICSB).

\section{References}

1 A. A. Jitariu, A. M. Cimpean, D. Ribatti and M. Raica, Triple negative breast cancer: the kiss of death, Oncotarget, 2017, 8, 46652-46662.

2 G. Bianchini, J. M. Balko, I. A. Mayer, M. E. Sanders and L. Gianni, Triple-negative breast cancer: challenges and opportunities of a heterogeneous disease, Nat. Rev. Clin. Oncol., 2016, 13, 674-690.

3 K. Oualla, H. M. El-Zawahry, B. Arun, et al., Novel therapeutic strategies in the treatment of triple-negative breast cancer, Ther. Adv. Med. Oncol., 2017, 9, 493-511.

4 J. Yao, P. Zhang, J. Li and W. Xu, MicroRNA-215 acts as a tumor suppressor in breast cancer by targeting AKT serine/threonine kinase 1, Oncol. Lett., 2017, 14, 1097-1104.

5 R. Garcia-Vazquez, E. Ruiz-Garcia, A. Meneses Garcia, et al., A microRNA signature associated with pathological complete response to novel neoadjuvant therapy regimen in triplenegative breast cancer, Tumor Biol., 2017, 39, 1010428317702899.

6 L. Lu, X. Mao, P. Shi, et al., MicroRNAs in the prognosis of triple-negative breast cancer: A systematic review and metaanalysis, Medicine, 2017, 96, e7085.

7 Y. Ito, A. Inoue, T. Seers, et al., Identification of targets of tumor suppressor microRNA-34a using a reporter library system, Proc. Natl. Acad. Sci. U. S. A., 2017, 114, 3927-3932.

8 S. Imani, X. Zhang, H. Hosseinifard, S. Fu and J. Fu, The diagnostic role of microRNA-34a in breast cancer: a systematic review and meta-analysis, Oncotarget, 2017, 8, 23177-23187.

9 B. D. Adams, V. B. Wali, C. J. Cheng, S. Inukai, C. J. Booth, S. Agarwal, D. L. Rimm, B. Győrffy, L. Santarpia, L. Pusztai, W. M. Saltzman and F. J. Slack, miR-34a Silences c-SRC to Attenuate Tumor Growth in Triple-Negative Breast Cancer, Cancer Res., 2016, 76(4), 927-939. 
10 X. Sui, X. Wang, W. Han, et al., MicroRNAs-mediated cell fate in triple negative breast cancers, Cancer Lett., 2015, 361, 812.

11 Y. Xin, M. Huang, W. W. Guo, Q. Huang, L. Z. Zhang and G. Jiang, Nano-based delivery of RNAi in cancer therapy, Mol. Cancer, 2017, 16, 134.

12 X. Deng, M. Cao, J. Zhang, et al., Hyaluronic acid-chitosan nanohybrids for co-delivery of MiR-34a and doxorubicin in therapy against triple negative breast cancer, Biomaterials, 2014, 35, 4333-4344.

13 S. Wang, M. Cao, X. Deng, et al., Degradable hyaluronic acid/ protamine sulfate interpolyelectrolyte complexes as miRNAdelivery nanocapsules for triple-negative breast cancer therapy, Adv. Healthcare Mater., 2015, 4, 281-290.

14 M. Figini, R. Ferri, D. Mezzanzanica, M. Bagnoli, E. Luison, S. Miotti and S. Canevari, Reversion of transformed phenotype in ovarian cancer cells by intracellular expression of anti folate receptor antibodies, Gene Ther., 2003, 10, 1018.

15 Y. Chen, O. Tezcan, D. Li, N. Beztsinna, B. Lou and T. Etrych, Overcoming multidrug resistance using folate receptortargeted and $\mathrm{pH}$-responsive polymeric nanogels containing covalently entrapped doxorubicin, Nanoscale, 2017, 9, 10404-10419.

16 M. M. El-Hammadi, A. V. Delgado, C. Melguizo, J. C. Prados and J. L. Arias, Folic acid-decorated and PEGylated PLGA nanohybrids for improving the antitumour activity of 5fluorouracil, Int. J. Pharm., 2017, 516, 61-70.

17 H. Li, C. Liu, Y. P. Zeng, et al., Nanoceria-Mediated Drug Delivery for Targeted Photodynamic Therapy on DrugResistant Breast Cancer, ACS Appl. Mater. Interfaces, 2016, 8, 31510-31523.

18 H. Jin, J. Pi, F. Yang, et al., Folate-Chitosan Nanohybrids Loaded with Ursolic Acid Confer Anti-Breast Cancer Activities in vitro and in vivo, Sci. Rep., 2016, 6, 30782.

19 A. Gupta, C. D. Kaur, S. Saraf and S. Saraf, Targeting of herbal bioactives through folate receptors: a novel concept to enhance intracellular drug delivery in cancer therapy, $J$. Recept. Signal Transduction Res., 2017, 37, 314-323.

20 L. Lai and A. S. Barnard, Modeling the thermostability of surface functionalisation by oxygen, hydroxyl, and water on nanodiamonds, Nanoscale, 2011, 3(6), 2566-2575.

21 M. Cao, X. Deng, S. Su, et al., Protamine sulfatenanodiamond hybrid nanohybrids as a vector for MiR-203 restoration in esophageal carcinoma cells, Nanoscale, 2013, 5(24), 12120-12125.

22 E. Gallardo, A. Navarro, N. Viñolas, et al., miR-34a as a prognostic marker of relapse in surgically resected nonsmall-cell lung cancer, Carcinogenesis, 2009, 30(11), 19031909.

23 T. C. Chang, E. A. Wentzel, O. A. Kent, et al., Transactivation of miR-34a by p53 broadly influences gene expression and promotes apoptosis, Mol. Cell, 2007, 26(5), 745-752.

24 N. Li, H. Fu, Y. Tie, et al., miR-34a inhibits migration and invasion by down-regulation of c-Met expression in human hepatocellular carcinoma cells, Cancer Lett., 2009, 275(1), 44-53.

25 L. Li, L. Yuan, J. Luo, et al., MiR-34a inhibits proliferation and migration of breast cancer through down-regulation of Bcl-2 and SIRT1, Clin. Exp. Med., 2013, 13(2), 109-117.

26 X. J. Li, Z. J. Ren and J. H. Tang, MicroRNA-34a: a potential therapeutic target in human cancer, Cell Death Dis., 2014, 5(7), e1327.

27 A. G. Bader, miR-34-a microRNA replacement therapy is headed to the clinic, Front. Genet., 2012, 3, 120.

28 P. S. Low, W. A. Henne and D. D. Doorneweerd, Discovery and development of folic-acid-based receptor targeting for imaging and therapy of cancer and inflammatory diseases, Acc. Chem. Res., 2007, 41(1), 120-129.

29 P. S. Ginter, P. J. McIntire, X. Cui, et al., Folate Receptor Alpha Expression Is Associated With Increased Risk of Recurrence in Triple-negative Breast Cancer, Clin. Breast Cancer, 2017, 17(7), 544-549.

30 B. M. Necela, J. A. Crozier, C. A. Andorfer, et al., Correction: Folate Receptor- $\alpha$ (FOLR1) Expression and Function in Triple Negative Tumors, PLoS One, 2015, 10(4), e0127133.

31 Z. Zhang, J. Wang, D. E. Tacha, et al., Folate receptor $\alpha$ associated with triple-negative breast cancer and poor prognosis, Arch. Pathol. Lab. Med., 2013, 138(7), 890-895.

32 J. Wu, G. Wu, L. Lv, et al., MicroRNA-34a inhibits migration and invasion of colon cancer cells via targeting to Fra-1, Carcinogenesis, 2011, 33(3), 519-528.

33 C. Zhao, Y. Qiao, P. Jonsson, et al., Genome-wide profiling of AP-1-regulated transcription provides insights into the invasiveness of triple-negative breast cancer, Cancer Res., 2014, 74(14), 3983-3994. 